\title{
Effect of intraocular lens implantation on combined extracapsular cataract extraction with trabeculectomy: a comparative study
}

\author{
R NEUMANN, M ZALISH, AND M OLIVER \\ From the Department of Ophthalmology, Kaplan Hospital, Rehovot, and the Hebrew University-Hadassah \\ Medical School, Jerusalem, Israel
}

SUMMARY In an attempt to assess the effect of posterior chamber intraocular lens (IOL) implantation on the outcome of combined extracapsular cataract extraction (ECCE) and trabeculectomy we compared 23 eyes subjected to ECCE and trabeculectomy with 23 eyes subjected to a triple procedure - that is, with the addition of IOL implantation. The results showed that IOL implantation did not have a detrimental effect on postoperative IOP reduction, gain in visual acuity, or needs for antiglaucoma medication. The incidence of anterior chamber reactions consisting of the development of posterior synechiae and fibrin formation was significantly higher when IOL had been implanted. However, the fibrin was generally absorbed within 14 days and the posterior synechiae did not occlude the visual axis. We conclude that IOL implantation should be included in these combined operations with the object of rehabilitating visual function.

Combined surgery for cataract and glaucoma has been performed with increasing frequency over the past decade. As the geriatric population increases, there is a growing number of candidates for this type of surgery. Another factor is the high success rate reported for procedures combining cataract extraction and trabeculectomy. Once the intracapsular method of cataract extraction had been widely replaced by the extracapsular technique, it seemed that the logical next step was to introduce a posterior chamber intraocular lens (IOL) at the same time with the object of rehabilitating phakic vision. Successful results of these triple procedures have been reported elsewhere. ${ }^{12}$ However, so far as we are aware there are not yet any comparative studies assessing the advantages and disadvantages of including IOL implantation in operations combining extracapsular cataract extraction (ECCE) and trabeculectomy.

In the present study we compare a series of 23 eyes subjected to ECCE and trabeculectomy in combination with IOL implantation with a series of 23 eyes subjected to ECCE and trabeculectomy only. The effects of including IOL implantation were assessed by comparing improvement in visual acuity, intraCorrespondence to $\mathrm{Dr} \mathrm{R}$ Neuman, Eye Department, Kaplan Hospital, 76100 Rehovot, Israel. ocular pressure (IOP) reduction, and immediate as well as later postoperative complications in the two series.

\section{Materials and methods}

The combined procedure of cataract extraction and trabeculectomy has been performed in our department since 1976. Early in 1985 we began to carry out triple procedures - that is, IOL implantation in addition to ECCE and trabeculectomy - and we have since included IOL implantation in all such operations except where specifically contraindicated, for example, in patients with high myopia, advanced proliferative diabetic retinopathy, or uveitis.

Two groups, each consisting of 23 patients, were studied. Each patient in the first group had undergone a combined procedure of ECCE and trabeculectomy, while those in the second group had undergone the triple procedure - that is, with the addition of IOL implantation. Each group comprised the last 23 consecutive cases to have undergone either the double or the triple procedure at the time of study; patients with high myopia, proliferative diabetic retinopathy, or uveitis were excluded.

The mean postoperative improvement in visual 
acuity and reduction in IOP were compared in the two groups. In each patient improvement in visual acuity was measured in terms of the difference in the number of lines on the Snellen chart read before and after the operation. If the preoperative visual acuity was poorer than $6 / 120$, one unit was added to the result, so that an improvement in visual acuity was indicated by a higher score. Each patient was examined with optical correction after refraction.

The mean preoperative IOP in each case was taken as the mean value obtained from the patient's last diurnal pressure curve recorded preoperatively. If no such curve had been recorded for more than three months prior to surgery, the average value of the four last consecutive IOP measurements prior to the operation was used. The mean postoperative IOP corresponded either to the mean of a diurnal pressure curve measured after surgery or, where this was unavailable, to the average value of the last four postoperative IOP measurements recorded in the patient's file. The postoperative reduction in IOP was obtained by simple subtraction.

The amount of antiglaucoma medication before and after surgery was compared in the two groups. Also compared were the incidence of immediate and later surgical complications, including anterior chamber reactions, anterior chamber depth, and formation of posterior synechiae.

The mean period of follow-up was 10.9 months (range 5-18 months) in patients who underwent the combined procedure of ECCE and trabeculectomy, and 3.8 months (range $2-10$ months) in those who underwent the triple procedure.

\section{PROCEDURES}

Cataract extraction was performed under local or general anaesthesia, depending on our prior assessment of the patient's ability to comply with the instructions of the surgical team during the operation. Local anaesthesia was used whenever possible. Following peritomy of $160-180^{\circ}$ and conjunctival reflection, a $3 \times 3 \mathrm{~mm}$ limbus-based scleral flap was cut to the desired depth and usually at the 12.00 o'clock position. A corneoscleral groove over 160 $180^{\circ} \mathrm{C}$ and two preplaced sutures were prepared on both sides of the scleral flap. A small opening was then made in the anterior chamber, usually close to the scleral flap, and anterior capsulotomy was carried out by the can-opening technique. A corneoscleral incision was made along the groove, and directed slightly towards the cornea at the site of the scleral flap. The lens nucleus was expressed and the preplaced sutures were then tied with a slip or final knot, depending on whether IOL implantation was planned or not. The lens cortex was aspirated by a Kelman aspirator or a McIntyre syringe. In patients receiving an IOL the temporary slip knots were opened and the IOL was implanted in the posterior chamber. An intraocular lens of the modified J loop type was used in each case. The corneoscleral incision was closed, first temporally and then nasally, by means of $10-0$ Ethilon sutures.

Following wound closure on both sides of the scleral flap a piece of tissue from the trabeculum area $(2 \times 2.5 \mathrm{~mm})$ was resected, iridectomy was performed at the site of the trabeculectomy, and the scleral flap was sutured with 4 or 6 separate $10-0$ nylon sutures. In eyes in which the anterior chamber was found to be shallow the irrigation aspiration procedure was followed by the injection of sodium hyaluronate (Healonid) in order to reform the anterior chamber. At the completion of the operation the anterior chamber was always well formed. The conjunctival flap was placed back in position and sutured with two single 9.0 braid silk sutures.

\section{Results}

Table 1 presents the clinical characteristic of the two groups studies, namely, the 23 patients who underwent the triple procedure of ECCE, trabeculectomy, and IOL (group A) and the 23 who underwent ECCE

Table 1 Clinical findings

\begin{tabular}{|c|c|c|c|c|c|c|c|c|}
\hline \multirow[t]{2}{*}{ Surgical procedure } & \multirow[t]{2}{*}{$\begin{array}{l}\text { No of } \\
\text { eyes }\end{array}$} & \multirow[t]{2}{*}{$\begin{array}{l}\text { Mean age } \\
(y r)\end{array}$} & \multirow[t]{2}{*}{$\begin{array}{l}\text { Male/ } \\
\text { female }\end{array}$} & \multicolumn{3}{|c|}{$\begin{array}{l}\text { Open angle glaucoma } \\
\text { Primary } \quad \text { Secondary }\end{array}$} & \multirow[t]{2}{*}{$\begin{array}{l}\text { Angle closure } \\
\text { glaucoma }\end{array}$} & \multirow{2}{*}{$\begin{array}{l}\text { Mean } \\
\text { follow-up } \\
\text { (months) }\end{array}$} \\
\hline & & & & Simple & $\begin{array}{l}\text { Pseudo- } \\
\text { exfoliation }\end{array}$ & Pigmentary & & \\
\hline \multicolumn{9}{|l|}{ Group A } \\
\hline $\mathrm{ECCE}+\mathrm{IOL}+$ trabeculectomy & 23 & $\begin{array}{l}72 \cdot 7 \\
(61-83)\end{array}$ & $10 / 13$ & 10 & 4 & 2 & 7 & $3 \cdot 8$ \\
\hline \multicolumn{9}{|l|}{ Group B } \\
\hline $\mathrm{ECCE}+$ trabeculectomy & 23 & $\begin{array}{l}73 \cdot 9 \\
(58-86)\end{array}$ & $12 / 11$ & 6 & 10 & - & 7 & $10 \cdot 9$ \\
\hline
\end{tabular}


Table 2 Mean visual acuity improvement and mean IOP reduction after surgery

\begin{tabular}{lll}
\hline Surgical procedure & $\begin{array}{l}\text { Mean improvement in } \\
\text { visual acuity } \\
\text { (units) }\end{array}$ & $\begin{array}{l}\text { Mean pressure } \\
\text { reduction } \\
(\mathrm{mmHg})\end{array}$ \\
\hline $\begin{array}{l}\text { Group A } \\
\text { ECCE+IOL+ } \\
\text { trabeculectomy } \\
\begin{array}{l}\text { Group B } \\
\text { ECCE+trabeculectomy }\end{array}\end{array}$ & $3.13 \pm 0.49$ & $6.27 \pm 0.97$ \\
\hline
\end{tabular}

and trabeculectomy only (group B). The mean ages in the two groups were similar and there were no significant sex differences between them. Sixteen patients in each group suffered from open angle glaucoma (primary and secondary). The numbers of patients with angle closure glaucoma, pseudoexfoliation glaucoma, and pigmentary glaucoma are indicated in the table.

A comparison of the operative results in terms of mean improvement in visual acuity and mean reduction in IOP shows no differences between the two groups (Table 2).

Table 3 summarises the incidence of postoperative complications. Anterior chamber reaction was significantly higher in patients of group $\mathrm{A}$, where 11 eyes showed fibrin formation on the surface of the

Table 3 Number of eyes with postoperative complications

\begin{tabular}{llll}
\hline Surgical procedure & Fibrin & Shallow chamber & Iris retraction* \\
\hline $\begin{array}{l}\text { Group A } \\
\text { ECCE+IOL+ }\end{array}$ & & & \\
trabeculectomy & 11 & 6 & 13 \\
$\begin{array}{l}\text { Group B } \\
\text { ECCE + } \\
\text { trabeculectomy }\end{array}$ & 5 & 5 & 7
\end{tabular}

*This refers to positioning of the iris behind the IOL, usually sectorial lens and iris as compared with only five in group B. The fibrin was readily absorbed within 14 days following local steroid treatment. Thirteen eyes of group A developed posterior synechiae and iris retraction, leading to anterior positioning of the lens in front of the iris, usually sectorial. Posterior synechiae developed in only seven eyes of group B, and they did not occlude the visual axis in either group. Shallow anterior chamber was observed in about one-quarter of the patients in each group, usually on the first or second day after surgery, but deepened within seven days in each case.

Table 4 shows the number of eyes receiving various types of antiglaucoma medication before and after surgery. It can be seen that surgery was followed by a substantial drop in the number of patients requiring such medication, with no significant differences between the groups. It should be noted that four patients from group A were excluded from this assessment because they continued to receive full treatment for reasons not relevant to the operation outcome: two of them did not understand our instructions to stop taking medication, while two were instructed to continue with medication because of high pressure in the fellow eye.

\section{Discussion}

Both glaucoma and cataract are most often found in geriatric populations. A considerable proportion of glaucomatous patients show cataractous changes as well, usually - but not always - of the nuclear sclerotic type.

We consider three groups of patients with cataract and glaucoma as candidates for combined surgery.

Firstly, those in whom glaucoma cannot be controlled despite maximum tolerable medication, and in whom a cataract severely impairs vision. Combined surgery is clearly indicated in such cases.

Secondly, those in whom cataract extraction is clearly indicated and in whom glaucoma is controlled

Table 4 Number of eyes receiving pre-and postoperative antiglaucomatous medication

\begin{tabular}{|c|c|c|c|c|c|c|c|c|c|}
\hline & \multicolumn{2}{|c|}{$\begin{array}{l}\text { Acetazolamide+ } \\
\text { eye drops* }\end{array}$} & \multicolumn{2}{|c|}{ Eye drops only* } & \multicolumn{2}{|c|}{$\begin{array}{l}\text { Eye drops: } \\
\text { one drug regiment }\end{array}$} & \multicolumn{2}{|c|}{ Free from treatment } & \multirow[t]{2}{*}{$\begin{array}{l}\text { Total no. } \\
\text { of eyes }\end{array}$} \\
\hline & Before & After & Before & After & Before & After & Before & After & \\
\hline $\begin{array}{l}\text { Group } A \\
\ddagger E C C E+I O L+ \\
\text { trabeculectomy }\end{array}$ & 15 & 3 & 7 & 1 & 0 & 5 & 0 & 10 & 19 \\
\hline $\begin{array}{l}\text { Group B } \\
\text { ECCE + trabeculectomy }\end{array}$ & 12 & 3 & 7 & 1 & 4 & 5 & 0 & 14 & 23 \\
\hline
\end{tabular}

*Pilocarpine $2 \%$. adrenaline $1 \%$ (Eppy), and timolol $1 / 2 \%$ in various combinations.

†Either pilocarpine or timolol.

$\ddagger$ Not included here among the patients receiving postoperative medication are two patients who continued to take medication through misunderstanding instructions and two who continued to take acetazolamide because of uncontrolled IOP in the fellow eye. 
by intensive therapy including carbonic anhydrase inhibitors. While surgical intervention for glaucoma would not normally be recommended for these patients, the fact that they must be operated on for cataract raises the possibility of adding a filtration procedure at the same time. A number of advantages are to be gained from this. (1) Antiglaucoma medication can then be reduced, thus improving compliance on the part of the patient. (2) A filtration procedure as an adjunct to cataract extraction may avert the IOP increase which is known to follow cataract surgery in the early postoperative days. ${ }^{3}$ This increase is especially hazardous for severely damaged optic discs. (3) The possible need for a second operation is avoided. Reoperation, in addition to imposing stress on elderly and infirm patients has certain specific drawbacks. For example, the lens must be removed through a corneal incision or at a section of the limbus at a distance from a future possible trabeculectomy site. It is our experience that these operations are less successful than with the regular method. We are impressed that these patients in whom glaucoma is controlled with intensive medication tend to have a raised IOP and to experience loss of glaucoma control in time. We therefore tend to favour combined surgery for these patients.

Thirdly, those in whom glaucoma surgery is indicated because maximum tolerable therapy does not control IOP levels, and a developing cataract has already caused partial impairment of the visual acuity. Although cataract extraction would not normally be recommended at this stage, the proposed filtering surgery for the glaucoma is likely to aggravate the cataractous changes in these patients. Reoperation for cataract after a filtering operation may inadvertently cicatrise a well-formed filtration bleb even when the cataract is removed through a corneal incision. Moreover, having the cataract removed allows improved follow-up and monitoring of the glaucoma by examination of the optic disc and visual field unhampered by opaque media. We would therefore advocate the combined procedure for this group of patients as well. It should be noted that IOL implantation is strongly recommended in this group of patients because their visual acuity prior to surgery is fairly good. Failure to implant an IOL would seriously hamper the rehabilitation of their visual function.

The purpose of this study was to clarify whether the outcome of the combined operation might be detrimentally affected by the addition of a third procedure, namely IOL implantation for restoration of phakic vision. Satisfactory results have been reported for the combined procedure without IOL implantation. ${ }^{4}$ In our study the inclusion of an IOL in the procedure was evaluated in terms of the following criteria: (a) improvement of VA; $(b)$ reduction of IOP; $(c)$ anterior segment complications; $(d)$ reduction of medication after surgery.

We therefore compared a group of 23 eyes that underwent the triple procedure with a similar group that underwent only ECCE and trabeculectomy. About half of the patients in each group were poor candidates for visual acuity improvement, mainly because of age related macular degeneration (ARMD) and severely damaged optic disc. This may explain the rather modest levels of mean improvement in visual acuity found in both groups (3.5 lines of the Snellen chart). It should be pointed out that, whenever the posterior segment was healthy, the visual acuity improvement was up to 7 lines on the Snellen chart. Despite the lack of difference between the groups with regard to visual acuity improvement, there is unanimous agreement that the restoration of phakic vision through IOL implantation is of great advantage to the patient.

The degree of IOP reduction was almost the same in the two groups (Table 2). These values become more meaningful when examined in the light of preand postoperative medical therapy. As Table 4 shows, 14 patients who did not receive IOL and 10 patients who did were relieved of all antiglaucoma medication after surgery. Only three patients in each group continued to require treatment with carbonic anhydrase inhibitors.

Eleven patients in whom the IOP was controlled before operation were nevertheless operated on for other reasons such as their poor tolerance to antiglaucoma therapy. None of them required heavy medication after surgery.

The number of patients suffering postoperative anterior chamber reactions differed in the two groups. Eleven of the eyes with IOL implants and five without implants developed fibrin in the anterior chamber within a few days after surgery. In all of them the fibrin was completely absorbed within about two weeks. More than half (13/23) of the eyes with IOL and only seven eyes without it developed iris retraction with posterior synechiae. Since the iris retraction did not block the visual axis in any of these eyes, it did not reduce the final visual acuity.

These results show that in both groups there was a relatively high degree of postsurgical reactions in the anterior chamber. It should be remembered that these patients had been treated for long periods of time, most of them for years, with pilocarpine and other drugs for glaucoma. It is a common experience that prolonged periods of treatment with miotic drugs render the iris atrophic and prone to the formation of posterior synechiae. Thus, anterior segment surgery in those patients is often accompanied by pupillary distortion. Pupillary irregularity 
is also due to the unresponsiveness of the iris musculature to the commonly used midriatic solutions during and after surgery. The presence of a foreign body such as an IOL in these eyes probably enhances the reactions mentioned above to a still higher degree. This explains the higher percentage of fibrinous exudate, iris retraction, and posterior synechiae found in the pseudophakic patients (Table 3 ). No significant difference was found in the postoperative shallowing period of the anterior chamber between the groups. We therefore do not consider this condition to be a cause of the pupillary distortions finally resulting.

A shallow anterior chamber was observed in the immediate postoperative period in six eyes with and five without an IOL, lasting for not more than one week. In no case was the anterior chamber flattened completely.

Other authors have reported that posterior chamber implantation of IOL concomitantly with ECCE in glaucomatous eyes has no effect on the incidence of postoperative IOP elevation. ${ }^{3}$ This is in agreement with our present findings that the inclusion of IOL implantation in the combined procedure of ECCE and trabeculectomy did not adversely affect the average reduction in IOP. There was also no detrimental effect on the postoperative medication regimen or the visual acuity improvement.

It is well known that IOL implantation offers the best chance of restoring vision, even in patients with ARMD or glaucomatous optic nerve damage. Postoperative complications, although more common with IOL implantation, did not affect the final results. We therefore conclude that combined surgery for glaucoma and cataract should not normally be considered a contraindication for IOL implantation unless there are other specific contraindications, such as uveitis, high myopia, or advanced proliferative diabetic retinopathy.

\section{References}

1 Percival SPB. Glaucoma triple procedure of extracapsular cataract extraction, posterior chamber lens implantation, and trabeculectomy. Br J Ophthalmol 1985; 69: 99-102.

2 Ohanesian RV, Kim EW. A prospective study of combined extracapsular cataract extraction, posterior chamber lens implantation, and trabeculectomy. Am Intraocular Implant Soc J 1985; 11: $142-5$.

3 Savage JA, Thomas VJ, Belcher CD III, Simmons JR. Extracapsular cataract extraction and posterior chamber intraocular lens implantation in glaucomatous eyes. Ophthalmology 1985; 12: 1506-16.

4 Shields MB. Combined cataract extraction and glaucoma surgery. Ophthalmology 1982; 89: 231-7.

Accepted for publication $28 \mathrm{July} 1987$. 